\title{
Les logiques du multiculturalisme dans les sociétés multinationales : une analyse des discours \\ canadiens
}

The Logics of Multiculturalism in Multinational Societies: An Analysis of

Canadian Discourses

Las lógicas del multiculturalismo en sociedades multinacionales: un análisis de los discursos canadienses

\section{Elke Winter}

\section{OpenEdition}

Journals

Édition électronique

URL : https://journals.openedition.org/remi/4827

DOI : 10.4000/remi.4827

ISSN : $1777-5418$

Éditeur

Université de Poitiers

Édition imprimée

Date de publication : 1 décembre 2008

Pagination : 7-29

ISBN : 978-2-911627-50-7

ISSN : 0765-0752

\section{Référence électronique}

Elke Winter, «Les logiques du multiculturalisme dans les sociétés multinationales: une analyse des discours canadiens », Revue européenne des migrations internationales [En ligne], vol. 24 - n³ | 2008, mis en ligne le 01 décembre 2011, consulté le 15 avril 2022. URL : http://journals.openedition.org/ remi/4827 ; DOI : https://doi.org/10.4000/remi.4827 


\title{
Les logiques du multiculturalisme dans les sociétés multinationales : une analyse des discours canadiens
}

\author{
Elke WINTER*
}

Au milieu des années 1990, des chercheurs canadiens développent une distinction importante entre les nations minoritaires et les minorités dites « ethniques » issues de l'immigration. Les deux concepts les plus connus étant la « diversité profonde » de Charles Taylor (1993) et la «culture sociétale » proposée par Will Kymlicka (1995). Ainsi, le premier souligne la « diversité profonde » vécue par les Québécois francophones et les Peuples autochtones ; ces deux groupes étant des Canadiens à travers leurs identités nationales particulières. En revanche, pour Taylor, les autres Canadiens, dont ceux issus de l'immigration, sont caractérisés par une diversité de «premier niveau ». Ils sont d'abord Canadiens et ensuite d'origine italienne, ukrainienne, britannique... ; leur identité ethnique collective particulière n'étant pas de l'ordre de la qualification « nationale ». Par ailleurs, dans la théorie libérale de Kymlicka, la «culture sociétale » permet aux individus de choisir leur vie par rapport à un horizon culturel significatif. Il assure que les minorités nationales comme les Québécois francophones et les Peuples autochtones ont développé, au cours de leur histoire, une «culture sociétale » absente chez les autres immigrants et leurs descendants ; ces derniers poursuivant, en premier lieu, un objectif d'intégration dans la société d'accueil.

Outre leur valeur théorique intrinsèque, les contributions de Taylor et de Kymlicka clarifient aussi la grande confusion conceptuelle qui entoure les minorités ethniques nationales et celles issues de l'immigration. Car au-delà de leurs divergences, les deux auteurs proposent la même conclusion : les minorités nationales sont à distinguer des groupes d'immigrants et méritent d'autres droits à la reconnaissance

* Assistant Professor, Department of Sociology and Anthropology, University of Ottawa, elke.winter@uOttawa.ca

[La rédaction remercie Yann Scioldo-Zürcher pour la relecture de cet article] 
collective, dont une plus grande autonomie institutionnelle. D'un point de vue sociologique, cette conclusion normative devrait toutefois rester ouverte à la négociation. Cependant, il est vrai que ces deux minorités se distinguent non seulement par leur degré d'organisation institutionnelle, moins achevé chez les groupes ethniques issus de l'immigration (Breton, 1964) mais aussi, par le type de leurs revendications, davantage orientées vers l'autonomie politique chez les minorités nationales. Bien que les propositions de Taylor et de Kymlicka aient provoqué de nombreuses critiques, la distinction entre groupes ethniques et groupes nationaux qu'ils opèrent a largement été acceptée. Mais, malgré leur grande influence, il existe une lacune inhérente à leurs théories : l'absence d'une analyse relationnelle entre ces deux types de mouvements sociaux et la question non abordée des politiques destinées à les accueillir.

Au Canada, le «vide épistémologique »sur les rapports entre les groupes nationaux et ethniques ainsi que les droits demandés par et accordés à ces groupes est doublement étonnant. D'une part, il s'agit-là d'une entorse fondamentale à la manière dont le multiculturalisme canadien a été conçu et discuté lors de sa mise en œuvre dans les années 1970 (McRoberts, 1997). D'autre part, la diversité à trois niveaux, entre la nation minoritaire québécoise, l'intégration multiculturelle des immigrés et l'autogouvernance des peuples aborigènes, est devenue un trait caractéristique de l'identité nationale pancanadienne (Kymlicka, 2003). Ainsi, ne conviendrait-il pas de savoir comment les différentes facettes de cette diversité interagissent sur le plan logique et discursif $^{1}$ ? En Europe également, d'autres chercheurs déplorent une « rupture analytique radicale entre les demandes que formulent les deux types de mouvements pour le pluralisme culturel : les nations minoritaires d'une part et les groupes ethniques d'autre part » (Máiz et Requejo, 2005 : 2). Ainsi, et à titre d'illustration, il serait important de savoir comment le nationalisme flamand influe sur les revendications des organisations d'immigrés présentes en Belgique. Ou encore, si en Catalogne, la reconnaissance des droits linguistiques de la minorité hispanophone a un impact sur la façon dont les immigrants sont accueillis. Néanmoins, à ce jour, peu de recherches ont été effectuées dans cette perspective.

S'il est important de maintenir les différences conceptuelles entre les divers types de minorités, cet article soutient que l'on doit également étudier la façon dont leurs revendications s'influencent mutuellement. Après tout, en tant que nouveaux venus dans le domaine social et politique des sociétés établies, les immigrés sont perçus et classifiés par rapport aux divisions et catégories sociales existantes. Quelles que soient leurs revendications, il semble tout à fait logique que les groupes ethniques soient perçus et traités en fonction des compromis préexistants et qu'ils s'adaptent aux discours sociétaux, en cherchant à emprunter une partie de leur autorité et de leur légitimité aux mouvements qui font figure de prédécesseurs. Dès lors, les formes établies de compromis à l'égard des minorités anciennes, comme le multinationalisme,

1 Sur le plan institutionnel, au Canada, il y a en effet peu d'interaction entre les trois types de minorités. Leur reconnaissance est traitée dans des pièces législatives distinctes, elle est administrée par différents ministères et elle est l'objet de sections spécifiques de la Constitution. 
sont aussi susceptibles d'influer sur l'intégration et les revendications des immigrés plus récents. Ainsi, en s'inspirant du cas canadien, cet article vise à revitaliser le débat sur les relations existant entre le multinationalisme et le multiculturalisme ${ }^{2}$. Il se penche sur le discours majoritaire et examine comment les groupes ethniques issus de l'immigration et leurs revendications multiculturelles sont construits par rapport au nationalisme québécois. Il s'agira, tout d'abord, de dresser un panorama du cadre théorique et méthodologique existant. Nous y proposons également des scénarios sur la manière dont le multinationalisme historiquement établi peut influer sur la mise en œuvre des politiques multiculturelles. Nous examinerons ensuite les discours d'opinion tenus par deux journaux canadiens durant la décennie 1990 et aborderons les argumentations qui ont servi à légitimer ou à délégitimer le multiculturalisme au regard du dualisme anglophone/francophone existant.

\section{PARAMÈTRES THÉORIQUES ET MÉTHODOLOGIQUES DE L'ÉTUDE}

Pluraliste ou non, une identité nationale représente un « compromis culturel » historiquement acquis entre des groupes préexistants. Andreas Wimmer nous met ainsi en garde contre l'effet de pouvoir particulier qu'implique ce compromis culturel. Érigé en un ensemble de normes, classifications collectives et visions du monde qui engagent tous les membres d'une collectivité, il circonscrit toutefois le champ des représentations collectivement acceptées et « limite l'horizon des possibilités à l'intérieur duquel les individus peuvent débattre dans leur quête de pouvoir et de reconnaissance » (Wimmer, 2002 : 35). Or, un compromis culturel historiquement négocié privilégie, par définition, les individus et les groupes qui ont participé à sa mise en place. Dès lors, les groupes qui entrent dans l'arène politique à une étape ultérieure font face à une double difficulté : non seulement les accords existants ne leur réservent pas d'espace mais, pour faire valoir la justesse et la légitimité de leurs revendications, ils doivent aussi formuler leurs préoccupations en tenant compte des représentations collectives largement acceptées. «Any way that does not at least connect to the prevailing cultural compromise is not understood and quickly marginalized in public debates » (Wimmer, 2002 : 37). L'auteur nous rappelle combien le changement social suit les « sentiers» préétablis que représentent les cadres cognitifs et/ou les structures bureaucratiques. S'inscrivant dans la logique d'un compromis culturel et institutionnel donné, le changement social ne remplace jamais les réalités existantes avec quelque chose qui serait vraiment « inouï ». Il transforme de manière graduelle les visions et les divisions présentes. Il est vrai que des forces extérieures, comme la mondialisation ou les normes

2 Notre recherche a bénéficié d'une subvention du Conseil de recherches en sciences humaines du Canada. Une version antérieure de l'article a été présentée dans le cadre d'une conférence sur Immigration, Minorities and Multiculturalism in Democracies organisée en 2007 par l'initiative de recherche Ethnicity and Democratic Governance, Queen's University, Kingston (Ontario). Nous remercions Will Kymlicka, Angela Demian, Sophie Guérard de Latour, Chantal Lavallée et Rémi Léger pour leur aide et leurs suggestions précieuses. 
supranationales, ont un impact sur les traditions nationales et peuvent en changer le cours supposément «prédéterminé ». À cet égard, Christian Joppke constate une harmonisation, une universalisation et une dé-ethnicisation des lois d'immigration et d'acquisition de la nationalité en Europe (Joppke, 2007). Cependant, ce phénomène ne semble pas concerner les lois d'intégration. Au contraire, les nouveaux arrivés, qui seraient admis selon des critères « universels » de mérite (dans les cas des immigrants) ou de besoins (dans le cas des réfugiés), doivent, par la suite, s'insérer dans des contextes nationaux ou locaux particuliers (Fourot, 2006). Le concept de la glocalisation (Robertson, 1995) décrit bien cette tension inhérente entre les impacts externes de la mondialisation et la « dépendance au sentier », inhérente à chaque société.

Ainsi, les identités sociétales et nationales sont construites à l'intérieur des relations de pouvoir inter- et intranationales. Dans cette perspective, les tendances à la communalisation ethnique (Vergemeinschaftung) et à 1'association civique (Vergesellschaftung), selon les termes de Max Weber, caractérisent toute nation (Winter, 2004). Le type de pluralisme normatif qu'une collectivité met en œuvre dépend de négociations et de rapports de force complexes entre la majorité nationale et les groupes minoritaires (ces derniers étant définis en termes de pouvoir plutôt que de démographie). Le multinationalisme et le multiculturalisme peuvent ainsi être distingués comme les deux principales formes d'un pluralisme normatif. Le multinationalisme implique d'octroyer une certaine autonomie aux minorités linguistiques, culturelles, religieuses ou ethniques ayant été reconnues comme "nations minoritaires ». Ces dernières années, le nombre accru d'immigrants dans presque tous les États occidentaux industrialisés a conduit à l'émergence du multiculturalisme comme une forme particulière de pluralisme normatif visant à organiser les minorités ethniques d'origine immigrée et, à les intégrer dans la société majoritaire (Winter, 2007a).

Définir le multinationalisme et le multiculturalisme d'une façon sociologique, c'est-à-dire comme des relations de pouvoir et non pas comme de simples principes normatifs catégoriquement distincts, nous amène à croire que les divers types de pluralisme s'influencent mutuellement. Trois raisons y président.

Tout d'abord, tout compromis culturel multinational impose une structure idéologique et discursive qui peut faciliter ou bloquer les revendications multiculturelles. Le multiculturalisme est favorisé lorsqu'on reconnaît une similarité entre les types de différenciation ethnique/nationale qui ont déjà acquis une légitimité, et les nouvelles formes de groupes et d'identités minoritaires. Les Pays-Bas peuvent servir d'illustration : on a souvent affirmé que l'organisation initiale, reposant sur divers piliers de la société néerlandaise, a facilité l'élargissement de ce système aux minorités ethniques. Il s'est agi de représenter le multiculturalisme comme une extension des droits de la citoyenneté qui seraient «typiquement pluralistes » (Penninx et Schrover, 2002). Cependant, le multinationalisme préétabli peut aussi être indifférent ou préjudiciable à la mise en œuvre du multiculturalisme. En témoigne le cas de la Suisse, où les droits et la reconnaissance accordés aux quatre groupes linguistiques historiques n’ont pas été étendus aux minorités ethniques plus récentes (Pfaff-Czarnecka, 2004). 
En second lieu, le multinationalisme et le multiculturalisme s'influencent mutuellement dans le processus de leur affirmation sociale, car aucun compromis culturel établi dans une société ne reste indéfiniment stable. De ce fait, les droits à la reconnaissance obtenus ou délaissés par un groupe affectent l'équilibre de pouvoir ainsi que l'horizon des possibilités normativement acceptées dans la société. Le cas néerlandais peut encore ici servir d'exemple. La laïcisation et la « dé-piliarisation » récentes des groupes majoritaires chrétiens ne restent pas sans effet : peu à peu, les allochtones (minorités ethniques issues de l'immigration), surtout les musulmans, se trouvent les seuls à demander des droits multiculturels (Vasta, 2007).

Cela nous amène à la troisième raison qui explique l'interdépendance des différentes formes de pluralisme au sein d'une société. Les niveaux d'unité et d'accord entre les groupes préétablis sont souvent surestimés. Ainsi, dans l'ouvrage The Established and the Outsiders, Norbert Elias et John Scotson notent qu' " un groupe établi tend à attribuer au groupe extérieur, qu'il envisage comme un tout, les « mauvaises » caractéristiques du segment, «le pire » de ce groupe, de sa minorité anomique » (Elias et Scotson, 1994 : xix, [notre traduction]). En d'autres termes, la collectivité qui accueille n'est pas homogène et abrite des querelles internes. Deux scenari sont alors possibles. D'une part, le conflit entre les groupes préétablis peut avoir des conséquences négatives pour les immigrants nouvellement arrivés. C'est ce qu'on a souvent observé concernant les États-Unis : «once immigrants arrived in the country, whatever their national origin or race, they were ideologically positioned within the hegemonic bipolar white-black model of American society »(Ong, 1996 : 271). D’autre part, en cas de conflit intérieur et face à des compromis instables, les frontières établies entre le «nous » et le « eux» deviennent plus poreuses. Ainsi, un multinationalisme conflictuel peut permettre aux nouveaux venus de mieux négocier leur inclusion. La thèse de Pierre Anctil sur la « double majorité » montréalaise peut ici appuyer la démonstration (Anctil, 1984 : 450). Le chercheur observe qu' « en attirant à elle toute l'attention, la confrontation politique et juridique entre francophones et anglophones de souche, avait dégagé au Québec un espace socioculturel bien délimité, que les [immigrants] allophones avaient pu occuper seuls ».

En somme, les identités nationales, comme « compromis culturels préétablis », sont un point de départ nécessaire pour comprendre les transformations pluralistes d'une société. De plus, ces accommodements peuvent reposer sur des accords ou inversement, sur des conflits. Ainsi, nous pouvons développer quatre hypothèses sur l'impact potentiel du binationalisme sur le multiculturalisme. L'accord sur le multinationalisme dans les représentations publiques de « qui nous sommes » peut :

- Être insignifiant, voire préjudiciable, à la reconnaissance des nouveaux venus.

- Conduire à une extension des droits pluralistes.

- Rendre extrêmement difficile la reconnaissance des nouveaux venus

- Ou inversement, favoriser leur intégration comme des « contingent insiders ». 
Toutefois, comme aucune des deux situations, compromis multinational préétabli davantage harmonieux ou conflictuel, ne semble prédéterminer les relations entre le multinationalisme et le multiculturalisme, il convient de nous pencher sur une étude de cas spécifique.

Le Canada est édifiant à cet égard. Bien que officiellement promu depuis 1971, le multiculturalisme restait soit absent, soit négativement connoté dans les discours publics des années 1980 (Karim, 2002). Il s'imposa toutefois à la fin du siècle, comme une forme d'imaginaire social (Abu-Laban et Gabriel, 2002 ; Karim, 2002) en dépit de l'effritement du soutien institutionnel en faveur du multiculturalisme et de la confrontation entre les deux «nations fondatrices », linguistiquement définies, ayant conduit le pays au bord de la scission en 1995. Y aurait-il un rapport entre le nationalisme québécois et l'acceptation croissante du multiculturalisme au Canada anglais $^{3}$ ?

Notre argumentation s'appuie sur deux types de matériaux. D'une part, nous avons construit une revue extensive de la littérature. D'autre part, nous avons effectué une analyse qualitative de discours de presse ${ }^{4}$. Par une recherche de « mots-clés » dans les répertoires électroniques (tels que Québec, Canada, multiculturalisme, diversité), 350 articles des journaux Globe and Mail et Toronto Star publiés à Toronto entre le $1^{\text {er }}$ janvier 1992 et le 31 décembre 2001 ont été sélectionnés. Après avoir été codés et catalogués, 123 articles d'opinion ont été soumis à l'analyse critique de discours telle qu'elle a été élaborée par Fairclough (1992) et Wodak (1998). Cette démarche nous a permis d'analyser un corpus déterminé et d'y identifier les relations explicites et implicites liant le multiculturalisme canadien au nationalisme minoritaire du Québec. Nous avons volontairement sélectionné la ville de Toronto, supposément la plus multiculturelle du Canada et choisi des journaux à grand tirage, au lectorat principalement urbain et politiquement situé au centre et au centre-gauche du débat politique. Toutefois, notre analyse qualitative ne saurait rendre justice à la complexité de la situation. D'une part, l'interdépendance entre le multinationalisme et le multiculturalisme se joue tant sur le plan idéologico-discursif que sur le plan organisationnel et structurel. Ce dernier n'est pas l'objet de notre analyse empirique. D'autre part, dans un pays qui englobe des contextes culturels, socio-économiques, géographiques et politiques extrêmement divers, les représentations de la relation entre le multinationalisme et le multiculturalisme sont multiples. Nous ne pouvons pas ici tenir compte de cette diversité de perspectives. Si nous ne prétendons pas produire des résultats représentatifs, en nous penchant sur les représentations des relations entre binationalisme et multiculturalisme dans un segment de la presse majoritaire torontoise nous proposons, en revanche, de mettre en lumière un rapport important oublié par la communauté scientifique et d'avancer quelques éléments théoriques pour améliorer sa compréhension.

3 Le Québec poursuit sa propre politique d'intégration pluraliste des immigrants (Pietrantonio et al., 1996).

4 Cette étude a bénéficié d'une subvention de recherche du Conseil de recherche en sciences humaines du Canada. 


\section{TYPES D'ARGUMENTATIONS DANS LA PRESSE CANADIENNE : ÊTRE OU NE PAS ÊTRE COMME « LE QUÉBEC »}

\section{L'extension du dualisme et ses limites}

Lorsque les membres de la Commission royale sur le bilinguisme et le biculturalisme ${ }^{5}$ ont conduit des audiences dans l'Ouest canadien, les immigrants d'origine autre que britannique ou française les ont interrogés sur ce qui préoccupe les Canadiens depuis : "If it is valuable for French Canadians to maintain their distinctive culture and identity, why is it not so for other groups? »Cette citation illustre la logique du multiculturalisme comme une extension du dualisme normatif au Canada. En s'insérant dans le compromis préétabli entre les deux « nations fondatrices » et, en s'appropriant le discours dominant sur la langue et la culture, les minorités ethniques d'origine européenne ont pu s'affirmer comme similaires aux Canadiens français et ont pu remettre en question leur position privilégiée ${ }^{6}$. En acquérant progressivement de l'influence politique, ces « autres groupes ethniques » sont devenus une « troisième force » dans la politique canadienne ${ }^{7}$. Leurs revendications ont conduit les membres de la Commission à «prendre en considération la contribution qu'ont apportée les autres groupes ethniques à l'enrichissement culturel du Canada », tout en réitérant leur perception du Canada comme pays biculturel (Commission royale pour le Bilinguisme et le Biculturalisme, 1969).

Dans ce processus politique postérieur aux recommandations de la Commission, les divers types de groupes ethnonationaux ont été « accommodés » de diverses façons, qui leur ont rarement donné satisfaction. Le Livre Blanc de 1969 proposait de remplacer la discrimination structurelle des Peuples aborigènes par une politique d'assimilation ; l'Acte officiel sur les langues transforma le dualisme national en bilinguisme officiel. En fin de compte, le biculturalisme et la « mosaïque » ethnoculturelle occidentale se traduisirent en multiculturalisme, érigé en politique d'État dès 1971. Dans son discours à la Chambre des communes, en octobre 1971, le premier ministre Pierre Trudeau déclara que « le multiculturalisme dans un cadre bilingue » n'était pas uniquement une politique officielle, mais aussi l'essence même de l'identité canadienne (House of Commons, 1971). Bien qu'en présence de deux langues officielles, il rappellait l'absence de culture officielle et assurait qu'aucun groupe ethnique ne devait avoir de préséance sur un autre. Cette politique marqua le passage de l'assimilationnisme au pluralisme normatif au Canada et se fondait sur le choix politique de passer « du binationalisme au bilinguisme et du bi-culturalisme au multiculturalisme » (Juteau, $2000: 18)$.

5 Établie par le Gouvernement fédéral en 1963, la Commission partait de l'hypothèse selon laquelle la société canadienne était composée de deux groupes linguistiques et culturels dont les rapports devaient être éclaircis.

6 Les minorités ethniques d'origine allemande et ukrainienne furent tout particulièrement actives et puissantes dans la revendication de leurs droits.

7 Le Sénateur ukrainien Paul Yuzyk introduisit ce terme dans son premier discours au Sénat, le 3 mai 1964 (Kelner et Kallen, 1974 : 33). 
Depuis lors, dans les discours dominants, la refonte de l'identité nationale canadienne, visant à mieux traduire l'apport multiculturel des populations minoritaires, est souvent représentée comme une transition du dualisme au pluralisme et s'est imposé comme partie intégrante du mythe fondateur canadien. En 1992, lors de sa dixhuitième visite au Canada, Elisabeth II, chef de l'État, assurait que « les deux communautés linguistiques fondatrices du Canada et ses peuples aborigènes ont constitué depuis une société multiculturelle enrichie par l'arrivée d'immigrants provenant de tous les coins du monde » $(\mathrm{G} / \mathrm{T}-1992-0702)^{8}$. « Notre histoire nous a préparés à être novateurs dans le monde moderne, où la diversité joue un rôle si important », affirma encore dix ans plus tard, dans son Discours du Trône, Adrienne Clarkson, ancien gouverneur général du Canada, (Government of Canada, 2004).

"From the moment of its birth, Canada had a complex, divided personality shared between French settlers and United Empire Loyalists fleeing the American Revolution. [...] By the 1890s, the Governor-General was assuring immigrants from Europe to the 'Last Best West' that their languages and religions would be respected under the umbrella of an English-speaking democracy on Canada's prairies. The conscious multiculturalism of the West was a natural extension of the Confederation of the centre. [...] Overlaying all this was the British connection [...]. By the 1940s, it was clear that many Canadians felt no such connection, and the decades since have allowed it to gently fade to pastels »(G-1999-0701).

La prolifération de la « diversité » positivement connotée (en opposition à la « différence » négativement connotée) est alors décrite comme une « extension » douce et naturelle de la « personnalité complexe » du Canada, « divisé entre les colonisateurs français et les loyalistes de l'Empire uni fuyant la Révolution américaine » (G-19990701). On constate une émergence « quasi naturelle » du multiculturalisme canadien, au travers de l'étrange rencontre entre le «fait français » et «l'américanité » (Winter, 2007b). La prolifération de la diversité ethnique s'épanouit d'abord sous la protection d'un « lien britannique » vaguement défini, et va ensuite de pair avec l'effritement apparemment pacifique de ce qui pourrait être appelé la «nation ethnique » britannique. Le conflit et la lutte, telles les crises de la conscription, le mouvement nativiste et l'internement des Canadiens originaires de «pays ennemis » sont minimisés, voire occultés. Ainsi, aux «profondes divisions farouchement partisanes entre les Français catholiques et les Britanniques protestants »(G-1999-0701), tout comme aux injustices passées à l'égard des Peuples aborigènes (Neu et Therrien, 2003), succèdent progressivement un présent et un avenir multiculturels harmonieux. La trajectoire du Canada comme nation renvoie à une évolution modèle partant d'une colonie plurielle « hargneuse » (G-1999-0701) pour aboutir à une société « heureuse » et normativement pluraliste.

8 Afin de maintenir l'authenticité des discours analysés, nous ne traduisons pas les citations en anglais, à l'exception des passages directement intégrés dans notre texte. Les guillemets doubles indiquent les citations. Les articles de presse que comporte notre échantillon sont identifiés par la première lettre du titre du journal, l'année de sa publication, le mois et le jour de sa publication. 
Cette vision provoqua de nombreux débats chez les francophones du Québec et les Peuples aborigènes. Se considérant comme des nations distinctes et non pas comme des groupes ethniques d'origine immigrée, ils rejetèrent le multiculturalisme comme une stratégie politique visant à coopter des Canadiens «tiers » dans une majorité canadienne-anglaise et blanche. Les commentateurs du Québec furent ceux qui émirent le plus d'objections à ce propos : « le Canada anglais a appris, au cours de son histoire de deux siècles, à compter sur l'immigration » pour établir sa suprématie sur les francophones canadiens. "Sans l'immigration [...] il n'y aurait pas eu de Canada anglais par sa population, sa langue et sa culture » (Bouthillier, 1997 : 15). En tant que telle, "la politique fédérale du multiculturalisme [...] ne peut avoir comme effet que de relativiser et de banaliser la place et l'importance de l'identité culturelle spécifique du Québec : au Canada, au mépris de l'histoire, celle-ci ne serait qu'un particularisme parmi d'autres, qu'un petit morceau d'une grande mosaïque » (Corbo, 1996 : 100). En adhérant au multiculturalisme et à la Charte canadienne des Droits et des Libertés, les Canadiens anglais ratifieraient « une alliance entre eux-mêmes et une très grande partie des immigrés. [...] Le rôle des combattants implique surtout de parler anglais, de forcer les gens à le faire, de reproduire fidèlement les attitudes traditionnelles des Anglais envers les Canadiens français, d'amener les immigrés à suivre la même politique » (Schwimmer, 1995 : 173).

Si certains ont soutenu que le multiculturalisme sapa la spécificité du Québec au sein du Canada (Rocher, 1971 ; McRoberts, 1997), nous ne devons pas non plus ignorer que le sujet canadien majoritaire n'est pas seulement anglophone, mais aussi blanc, européen, chrétien et mâle. Les femmes, les minorités racialisées, les peuples aborigènes et les minorités religieuses (de nos jours surtout musulmanes) continuent à lutter contre diverses formes de discrimination au Québec et ailleurs au Canada (Helly, 2004). Selon la loi canadienne, les Canadiens anglais et français bénéficient toujours de plus de droits collectifs que les autres minorités ethniques ${ }^{9}$. La vision de Charles Taylor (1993) sur les Québécois et la "diversité profonde » des Aborigènes a été tout particulièrement critiquée de la façon suivante :

"[It] relegate[s] the others to the status of second-class citizens just as it relegates questions of the representation of ethnic/racial minorities to a much lower rank of importance than the representation of [the French and English] charter groups »(Stasiulis, 1995: 212).

En effet, dans la plupart des sociétés, on peut observer une tendance à considérer que les droits des groupes majoritaires vont de soi. Constituant les piliers « normaux » de la société, ils restent souvent implicites. Ce n'est que lorsque les minorités formulent leurs revendications qu'il devient évident que «l'extension » des droits de la majorité aux minorités rencontre des limites clairement définies :

9 En principe, les Peuples autochtones bénéficient de plus de droits collectifs que les groupes d'immigrants, par exemple des droits de pêche, la création de Nunavut et l'Accord Nisga'a. S'il s'agit de démarches dans la bonne direction, beaucoup d'Autochtones au Canada sont trop marginalisés pour pouvoir vraiment bénéficier de leurs droits civiques. 
"No one rationally is going to suggest that Canada establish Islam's sharia law alongside the English common law, the Quebec Civil Code and whatever the nation's aboriginal justice systems evolve. Apart from anything else, it would not be practical. This illustrates private versus public culture and non-institutionalized versus institutionalized culture. Multiculturalism - for now, at any rate - does not mean that everyone's culture is on the same institutionalized footing as English, French and aboriginal cultures »(G-1994-0617).

Cette citation démontre que le bi, ou même le trinationalisme, n'aboutit pas automatiquement et de façon inconditionnelle aux droits multiculturels pour les immigrants ${ }^{10}$. Elle confirme également l'idée selon laquelle « le multiculturalisme n'a jamais vraiment remis en cause la position prééminente de la politique nationale organisée autour du clivage franco-anglais » (Stasiulis, 1995 : 203). Dans la précédente citation, le trinationalisme serait majoritaire et « universel ». Les remises en cause du statu quo sont rejetées au nom d'un argument «pragmatique », de la « rationalité » et de la distinction entre « la culture privée et la culture publique ». En construisant une différence fondamentale entre les institutions « canadiennes » historiquement consolidées et « la culture [privée] de chacun », l'extension des droits collectifs multiculturels se transforme d'un «problème public » en un «conflit privé » (Mills, 2000). L'extension « irrationnelle » des droits sous-tendant le passage du dualisme et/ou du trinationalisme vers le multiculturalisme est ainsi bloquée.

Si le bi- ou le trinationalisme au Canada ne conduit pas automatiquement à la prolifération des droits et de la reconnaissance, il offre néanmoins un modèle d'extension des droits des minorités. À nous d'analyser cette dynamique de plus près.

\section{Revendications de multiculturalisme}

Il faut admettre que les demandes de reconnaissance ne suivent pas toujours la logique du pluralisme. Les débats vifs continuent à opposer, y compris au Canada, ceux qui pensent que la cause des groupes ethniques est le mieux défendue au travers des droits collectifs différenciés et ceux qui considèrent qu'elle l'est par le biais de l'égalité individuelle. Dans les discours ici analysés, l'option d'une " canadianité sans trait d'union » au sein d'une " communauté imaginée », fidèle à sa promesse d'être une « camaraderie horizontale » (Anderson, 1991) sans divisions verticales qui épouseraient les délimitations ethnoculturelles, est souvent perçue comme un idéal utopique. Comme l'évolution historique du Canada repose sur l'ethnicité et comme « l'assimilation par la culture française ou britannique » (T-1995-0123) est rejetée, le multiculturalisme devient le « deuxième meilleur » choix pour les immigrants. Le multiculturalisme serait un cadre plus inclusif que les modèles alternatifs d'intégration nationale, comme le projet sociétal basé sur le libéralisme orthodoxe (l'individualisme) ou celui basé sur la reconnaissance de deux (voire de trois) nations (Stasiulis, 1995).

10 Dix ans plus tard (2004-2005), à Ontario, l'idée de la sharia sera sérieusement discutée, mais pas implémentée (Razack, 2007). 
Comme nous l'avons déjà constaté, la mise en œuvre du multiculturalisme au Canada a largement suivi la logique selon laquelle les «minorités méritent elles aussi d'être reconnues ». Cela apparaît également dans les articles que comporte notre échantillon de presse :

"Until 1971 biculturalism was acceptable to most. It was felt those who were neither French nor English did not bring anything worthwhile with them. Their culture was looked down on. They were forced to 'assimilate' into the French or British culture. If Bissoondath has his way, would he be able to persuade Quebecers that they should just be Canadians and not French Canadians? Is it realistic to ask them to do so? » (T-1995-0123).

Le romancier canadien Neil Bissoondath (1994), attaqué dans la citation cidessus, adhère au caractère binational du pays mais critique la particularisation multiculturelle de l'identité canadienne, y compris les programmes «d'action affirmative » destinés aux membres des «minorités visibles » ${ }^{11}$. Il est aussi intéressant de noter qu'il y a de nombreux exemples, à l'instar de la citation ci-dessus, dans lesquels le point de référence choisi ne renvoie pas aux droits majoritaires des Canadiens anglais, qui iraient de soi, mais plutôt aux droits minoritaires des Franco-québécois. La citation cidessus illustre ainsi l'ambiguïté permanente qui caractérise les discours relevés dans notre échantillon de presse : les Franco-québécois empêchent-ils la consolidation d'une identité canadienne sans trait d'union (ni française, ni britannique) ou représentent-ils l'exemple le plus puissant d'un désenchantement face à une identité canadienne qui n'est qu'en apparence « universelle » ? D'une part, le nationalisme minoritaire, notamment « la restrictive Loi 101 sur la langue française adoptée dans cette province », est dénoncé comme une «façon de bafouer les droits démocratiques de ceux qui immigrent au Québec »(T-1998-0223). De ce point de vue, l'accent sur le binationalisme est perçu comme un obstacle à l'extension des droits multiculturels : en « exigeant des enfants des nouveaux venus (notamment des minorités) de fréquenter les écoles françaises, le gouvernement du Bloc » ${ }^{12}$ est accusé de « créer d'énormes obstacles au multiculturalisme et à la diversité de notre nation » (T-1998-0223).

D'autre part, on affirme que « le problème du Québec ne représente qu'une des questions non réglées portant sur la manière dont nous vivons ensemble sur cette terre appelée le Canada »(T-1995-0829). Dans cette perspective, la «Charte des Droits, le bilinguisme, le multiculturalisme, les affaires autochtones et notre politique d'immigration qui évoluent » ne sont que des « réponses modérées » à la diversité ethnique qui ne cesse d'augmenter (T-1995-0829). Dans les deux cas, la représentation des droits des minorités repose sur l'idée de similitude entre les nations minoritaires

11 La catégorie de «minorités visibles » est une construction ambiguë. Les critères de la « visibilité » renvoient tantôt à la couleur de la peau (par exemple les Blacks), tantôt à l'appartenance culturelle et/ou religieuse (par exemple les Arabes et les Sud-Asiatiques), tantôt encore à la nationalité d'origine (par exemple les Chinois).

12 Il s'agit ici d'une confusion entre le Bloc québécois et le Parti québécois. 
(qui disposeraient davantage de pouvoir) et les groupes ethniques (qui seraient dotés de moins de pouvoir). Cette similitude sert également à défendre l'exigence de plus de droits minoritaires pour les immigrants :

"People who are not part of the ruling elite in this country do not feel that they fundamentally belong. The 'Two Founding Nations' speeches cannot capture the hearts and spirits of French Quebecers who do not have the sense of sharing power. Just as the myth of multiculturalism is no solace to immigrants confronted with Markham councillors threatening to leave town because so many Chinese are congregating there. [...] The separatist movement must be taken as a wake-up call for all Canadians. Its sentiments of alienation are not limited to French Quebecers»(T-1995-1016).

La lutte pour les droits et la reconnaissance de la minorité la plus puissante sur le sol canadien, c'est-à-dire la lutte des Franco-québécois, ne serait que la «partie visible de l'iceberg » de « l'aliénation » minoritaire. Contrairement à la critique du binationalisme, au profit des revendications multiculturelles, argumentation présentée au début de cette section, le nationalisme minoritaire est ici présenté comme le fer de lance du multiculturalisme. On l'utilise pour attirer l'attention sur la lutte de ceux qui sont moins puissants. Afin de promouvoir les droits de ces derniers, on construit une histoire permettant de conférer au syntagme "politique de l'identité » une ancienneté et une légitimité. On utilise la similitude entre les groupes ethniques et le Québec pour s'approprier une partie du pouvoir factuel et symbolique attribué au Québec francophone :

"[Critics of multiculturalism say] "identity politics" are new. This is rewriting history. The 1867 Constitution was all about recognizing identities - French and English, Catholic and Protestant, and the four original provinces - each maintaining its identity in a united Canada. One of our great strengths has been that we have been able to recognize identities and accommodate them. It is the failure to recognize one of these identities - Quebec's - that recently brought Canada to the edge » (T-1996-0104).

La citation ci-dessus pointe vers la légitimité des droits à la reconnaissance des minorités, tout en énonçant une menace implicite : « la non-reconnaissance des identités conduirait le Canada « au bord du désastre »; le séparatisme québécois étant un exemple concluant en ce sens. Par conséquent, il vaudrait mieux qu'on traite les demandes en faveur du multiculturalisme avec sérieux ! » Si ce type d'argumentation a aidé les minorités ethniques à contester la position privilégiée qui est le plus souvent attribuée aux nations minoritaires, construire une similitude entre les groupes ethniques et les nations minoritaires peut également avoir un effet néfaste. Dans la section qui suit, nous allons analyser la manière dont les références au Québec servent à justifier les rejets conservateurs du multiculturalisme.

\section{Rejets du multiculturalisme}

Tant que les demandes multiculturelles formulées par les minorités visent à l'intégration et non pas à la séparation, elles sont soit acceptées et insérées dans la logique d'un dualisme qui s'élargit (comme nous l'avons noté dans la section 
précédente), soit minimisées et intégrées dans la logique d'un dualisme qui s'effrite : "Perhaps it is time for us to allow our founding people, the French and the British, to rest in peace. They carved out a country, earned our respect and their place in our history, but they are dead »(T-1993-0321). En effet, une des stratégies potentielles permettant de contrecarrer les demandes de multiculturalisme consiste à dissoudre la tradition même du multinationalisme. Ce type de raisonnement repose sur l'idée libérale selon laquelle « l'État n'a pas à tenir compte de l'arbre généalogique de la nation » (T-1993-0321) et les Canadiens doivent « être personnellement responsables de leurs héritages culturels » (T-1993-0321). Ici, les minorités ethniques sont intégrées comme partie du « peuple » au sein d'un État libéral. Toutefois, leur qualité de membre reste conditionnelle, dépendant de leur propension à se dépouiller de leurs identités culturelles particulières :

\begin{abstract}
"Strong forces of nationalism are loose in the land outside Quebec. [...] They express themselves in the desire [...] to be one people in a liberal state, rather than a collection of hyphenated peoples in a vague confederation. (Many so-called ethnic Canadians feel this way.) [...] Many Canadian nationalists believe that official bilingualism and its fraternal twin, official multiculturalism, inhibit the growth of a coherent English-speaking nation. They want more emphasis in the public sphere on things we have in common, leaving the nourishment of differences to private lives. Can this be appreciated in francophone Quebec, which apparently feels the same way about its own society? »(G-1992-0111).
\end{abstract}

Ainsi, une similitude est construite entre le bilinguisme officiel et « son jumeau fraternel », le multiculturalisme. Le bilinguisme est vu comme une concession envers les Canadiens français. Le multiculturalisme est décrit comme concession envers les groupes ethniques. Les deux politiques seraient donc des invasions : elles « empêchent la consolidation d'une nation anglophone cohérente ». Cette situation suggère également que les groupes ethniques qui résident au Canada ailleurs qu'au Québec et les Canadiens français qui vivent au Québec appartiennent à deux ordres différents : tandis que les «Canadiens ethniques » doivent être intégrés au sein d'une nation anglophone en tant qu'individus, le «Québec francophone » est construit comme une entité séparée dotée de «sa propre société » au sein du Canada.

Compte tenu de la mise en question de la «neutralité culturelle » de l'État libéral dans la littérature académique, au début des années 1990, les demandes de droits multiculturels se consolidèrent. Ce mouvement est suivi d'une croissante opposition conservatrice à tous les types de "groupalité ». À cette époque, le Québec est souvent décrit comme la principale source de prolifération de la « différence » ethnique, négativement connotée, plutôt que de la "diversité » ethnique, positivement connotée (Winter, 2007b). En outre, au Canada anglais, le pouvoir destructeur du séparatisme québécois et le rôle pionnier des Canadiens français dans la contestation de l'identité canadienne-anglaise conduisirent à faire du Québec un symbole pour la fragmentation sociale. Dans cette situation, les demandes minoritaires suggérant que « la lutte du Québec pour la reconnaissance nous représente tous » s'avèrent contreproductives : les références au Québec francophone sont de plus en plus utilisées pour rejeter en bloc les revendications des minorités d'étendre le multiculturalisme : 
"It may seem outlandish to suggest that Anglo Canadian culture could become marginalized. It is still financially and commercially dominant, and remains on top politically. A precedent, though, has been set in Quebec. Until the late 1950s, the famed Anglo Scottish Ascendancy dominated the province's public life. Today, those 'White Rhodesians,' although still comparatively well-off, are scarcely more influential than are the various expatriate communities in cities like Paris, Rome, London » (T-1993-0228).

Cet extrait suggère qu'un multiculturalisme qui ne cesse d'augmenter produit en définitive une situation semblable à celle de la «Révolution tranquille » au Québec, où les Canadiens anglais furent «marginalisés » par la montée d'une classe moyenne francophone. La notion de «précédent » suggère qu'il y a une «preuve » que la culture canadienne-anglaise est menacée et que, ce qui s'est passé au Québec peut également arriver ailleurs au Canada ; bien que « la culture anglo-canadienne [...] reste dominante du point de vue financier et que, politiquement, elle l'emporte toujours ». Dans ce type d'allégations, on fait un parallèle entre les groupes ethniques et les nations minoritaires. Ainsi, que ce soit de façon explicite ou implicite, on suggère que « notre multiculturalisme peut se transformer en multinationalisme » (T-1993-0228). D'une part, les groupes ethniques sont de plus en plus décrits comme des «groupes diasporiques » qui « se soucient avec plus de passion des événements qui ont lieu dans leur pays natal que de ce qui se passe dans leur pays d'adoption » (T-1993-0228). D'autre part, le nombre croissant de Canadiens non blancs est présenté comme une conquête culturelle et linguistique du/sur le sol canadien :

"If an 'ethnic' community becomes larger than the European-descended community, it will grow more assertive. It will see no reason to defer to a culture that has fewer adherents than its own. It might change the local bylaw so that it can cut down as many trees as it wants. It might want offices and schools to close on its holidays and to remain open on holidays it doesn't celebrate. It might decide to make its language official and have it spoken in the legislature, placed on public signs, and compulsory in schools »(Stoffman, 2002 : 134-135).

Bien que l'Autre menaçant soit identifié comme non Européen, la situation qui est décrite fait allusion à l'expérience du Canada anglais avec le Québec et donc évoque la mémoire de luttes, négociations, «citoyenneté de second ordre » ainsi que la menace d'une séparation. Symbole puissant de l'effritement de la domination canadienne-anglaise, interprété comme une " désintégration sociétale », le Québec francophone est érigé en exemple terrifiant de ce vers quoi peut conduire le «vrai multiculturalisme » (Stoffman), en réponse à la diversité ethnique provenant de l'immigration. Dans certains cas, la similarité entre les groupes ethniques et le Québec est explicitement formulée ( « les groupes ethniques peuvent devenir comme le Québec»). Dans d'autres cas, d'une manière plus prudente, une similarité indirecte est construite au regard des résultats parallèles des deux types de demandes minoritaires :

"[M] ulticulturalism, along with other factors such as our passive acceptance until very recently that Quebecers had a unilateral right to decide whether to cease being Canadians, has made our common citizenship insubstantial or weightless » (T-1997-0219). 
Que la similarité soit directement ou indirectement posée, la logique d'ensemble reste la même : les groupes ethniques et les nations minoritaires sont quasi semblables ; le multiculturalisme est une simple extension du dualisme. Ce qui change dans ces représentations, par rapport à celles qui sous-tendent les demandes de multiculturalisme, c'est tout simplement la connotation normative du pluralisme (réduisant plutôt que consolidant l'égalité) et les résultats attendus (fragmentation sociétale plutôt qu'intégration).

\section{«Les minorités ethniques ne demandent pas l'autogouvernance »}

En réaction à ces reproches, les minorités ethniques ont réaffirmé leur loyauté à la nation canadienne. Néanmoins, pendant longtemps, leur discours ne fut pas entendu. Cette situation change dans la deuxième moitié des années 1990. Dans notre matériel empirique, on peut observer un changement discursif passant de «les minorités multiculturelles souhaitent/méritent les mêmes droits culturels que les francophones » à «les minorités multiculturelles ne formulent pas les mêmes revendications politiques que le Québec ». Dans cette dernière représentation des relations ethniques au Canada, on construit une différence fondamentale entre « le nationalisme minoritaire séparatiste » du Québec et un «pancanadianisme multiculturel » lié à la diversité ethnique issue de l'immigration :

"Those who blame multiculturalism and the lack of Canadian loyalty from ethnic communities need only look at the Parti Quebecois assessment of its recent attempt to break up Canada. One blamed the ethnic vote and another blamed the 'ravages of multiculturalism' for keeping Canada united. When the chips were down, the ethnic vote was 100 per cent pro-Canada » (T-1996-0104).

La comparaison avec les Franco-québécois, dont $60 \%$ votèrent en faveur de la séparation lors du referendum québécois de 1995 sur l'indépendance, permet aux minorités ethniques de se défaire du stigmate les accusant d'être catalyseurs de la fragmentation sociale. Les faits confirment cette situation. Pendant le référendum, $95 \%$ de la population non francophone du Québec vota en effet contre la séparation (Drouilly, 1997). Ce comportement de vote est interprété comme une consolidation de la place légitime des minorités ethniques au sein de la société pancanadienne. Il donne lieu à un nouveau type de représentation : les minorités ethniques ne sont plus des « conditional insiders » aux marges d'une nation dualiste. Elles sont plutôt décrites, et elles se décrivent elles-mêmes, comme des alliés multiculturels de la nation pancanadienne :

"[T]he biggest challenge to Canadian citizenship comes not from the new minorities but our oldest ones - the aboriginals and the English and the French. [...] 'National minorities,' in the words of Will Kymlicka [...] insist on nation-tonation negotiations in the 'multi-nation state' that is Canada. But immigrants Canadians by choice rather than the accident of birth make no such demands. They don't ask for self-government. They are not potentially secessionist; in fact, those in Quebec are in the forefront of fighting separatism »(T-2000-1105). 
Cette citation révèle que la redéfinition des revendications multiculturelles dans les discours majoritaires canadiens repose sur deux événements : le référendum de 1995 au Québec et la publication, la même année, de l'influente philosophie de la Citoyenneté multiculturelle de Will Kymlicka. La distinction que formule Kymlicka entre les droits octroyés aux nations minoritaires, aux peuples aborigènes et aux groupes ethniques d'origine immigrée fut négativement interprétée comme une façon d'établir une hiérarchie des droits en faveur des nations minoritaires et des peuples aborigènes. Pourtant, la citation ci-dessus montre qu'elle peut également être positivement utilisée pour séparer les groupes minoritaires d'origine immigrée des nations minoritaires qui nourrissent des intentions potentiellement sécessionnistes. Les groupes ethniques " ne demandent guère l'autogouvernance », ni l'autonomie politique. Selon Kymlicka (1995), leurs revendications de droits multiculturels devraient plutôt être interprétées comme une quête d'inclusion. Ainsi, « l'attachement des immigrés récents à leurs anciennes patries » «diminue[rait] rarement leur canadianité » (T-2000-1105). Ce sentiment «consolide » plutôt l'engagement des immigrés récents au Canada. En outre, bien que « les minorités les plus récentes» puissent souvent s'opposer dans des conflits globaux, dans le contexte canadien, elles « gardent une distance pacifique entre elles » (T-2000-1105). D'ailleurs, la distinction entre les «minorités nouvelles et anciennes » est approfondie :

"Newer minorities are not transplanting 'old country' troubles here, as the old ones did. Nor are they narrow regionalists. They strongly identified with Pierre Trudeau precisely because of his pan-Canadian vision (not, as some shoddy commentaries had it, because he 'let them' into Canada). Immigrants are less complaining and less demanding than the native-born »(T-2000-1105).

Cette citation illustre un nouveau courant des demandes minoritaires dans le contexte de la double majorité : les demandes ne reposent pas sur la similarité avec les groupes établis, mais plutôt sur l'affirmation de la différence : « les immigrants les plus récents surtout »(T-2000-1105) sont décrits comme "de meilleurs Canadiens », puisqu'ils seraient des «pancanadianistes » pacifiques qui « se plaignent moins et demandent moins » que les membres des soi-disant «nations fondatrices ». Ils ont « contribué à bâtir le Canada tel qu'il est - la meilleure nation au monde » (T-19980614) et ils l'ont « choisi » pour être leur « nouveau pays parce que le Canada est un lieu de vie beau, magnifique et très spécial » (T-2001-0424).

Nos données empiriques montrent que le refus d'être «piégé dans un ancien modèle » de diversité va de pair avec une valorisation croissante du « choix ». Cette vision très libérale du multiculturalisme conteste profondément l'idée selon laquelle « le sang est plus fort que l'eau ». Les immigrants d'aujourd'hui seraient plus dévoués que les groupes établis justement parce qu'ils sont, selon une citation déjà évoquée, « des Canadiens par choix plutôt que par accident de naissance » (T-2000-1105). Dans le contexte de la globalisation, où les États sont en compétition pour attirer des « immigrants sur mesure » hautement qualifiés (Simmons, 2004), le multiculturalisme devient un atout de la construction nationale plutôt qu'un handicap :

"We want more educated and wealthy immigrants to come to Canada. We do not take anymore the 'wretched refuse' (to quote from the Statue of Liberty). Are they going to embrace wholeheartedly the British and French culture in Canada or 
would they want to maintain some of their culture and traditions? The 'wretched refuse' would have happily embraced biculturalism, lock, stock and barrel " (T-1995-0123).

Ainsi, on affirme que «si les minorités deviennent mieux acceptées dans la société canadienne [...], avec le temps, les politiques d'équité dans le domaine de l'emploi deviendront superflues » (T-1995-0123). Les « nouveaux immigrants » n'auraient plus besoin d'être soutenus par leurs « communautés ethniques ». Ils souligneraient plutôt l'importance de "permettre à chaque Canadien de parler la langue de son choix, de vivre et de travailler tranquillement et en paix les uns avec les autres au sein d'une nouvelle nation constituée par des personnes de diverses cultures et d'horizons différents » (T-2001-0424). En effet, seules quelques voix minoritaires contenues dans notre échantillon de discours journalistiques revendiquent des droits collectifs (voir notamment T-1992-1104, T-1995-0811). Il est beaucoup plus courant de constater de la part des minorités des refus d'inspiration individualiste d'être traitées comme « des citoyens de second ordre» :

"Newcomers are [said to be] guests in the Canadian house and had better behave. This is obviously still true of refugees. But they constitute barely oneeighth of our annual intake of 200,000 immigrants. For most others, especially the well-educated and skilled in high-tech and information technology, coming to Canada is a contract; they trade their talents for membership in a great nation » (T-2000-1105).

Il s'agit bien ici de l'illustration de la tendance se dirigeant vers une conception de plus en plus libérale de la «construction nationale multiculturelle ». Cette citation nous rappelle également que notre échantillon de presse est constitué de discours majoritaires : alors que les immigrés éduqués peuvent « échanger leurs talents contre la qualité de membre d'une grande nation », les réfugiés non qualifiés restent des « invités » et doivent « bien se tenir ». La ligne de division ne sépare plus les groupes établis et les nouveaux venus, mais plutôt ceux qui peuvent et ceux qui ne peuvent pas «payer » leur qualité de membre de la nation.

\section{CONCLUSION}

Bien que la ville de Toronto n'a jamais été caractérisée par la présence d'une « double majorité » francophone et anglophone qui aurait concrètement influé sur "l'intégration sans assimilation » des groupes ethniques non britanniques et non français, à l'instar de ce qu'observe Anctil (1984) pour le cas montréalais, les discours de presse de l'édition torontoise du Globe and Mail et du Toronto Star font toutefois ressortir des relations intenses, diversifiées et changeantes entre le binationalisme et le multiculturalisme dans les représentations de la majorité canadienne.

Les discours d'opinion ici étudiés nous obligent tout d'abord à nuancer la représentation populaire d'un multiculturalisme comme étant la simple extension d'un multinationalisme. Ce dernier ne conduit ni automatiquement, ni entièrement, à une extension des droits multiculturels et à la reconnaissance des minorités. Tant que les 
membres de la majorité s'accordent sur leurs rôles et privilèges respectifs en tant que « fondateurs » de la nation, l'évolution du binationalisme au trinationalisme voire au multiculturalisme reste difficile et incomplet. Le multinationalisme, comme compromis, impose également une logique et une terminologie dans lesquelles les revendications doivent s'insérer (puisque tout le reste ne serait ni entendu, ni compris).

Que le multinationalisme circonscrive l'expression de la diversité légitime ne veut pas dire qu'il ne fournit pas de modèle pour l'extension des droits des minorités. Dans le contexte d'un multinationalisme officiel, les revendications en faveur (et de la part) des minorités multiculturelles ont tendance à suivre la logique de l'extension de la reconnaissance à d'autres groupes minoritaires. À cet égard, il est intéressant de noter que la formulation des demandes multiculturelles conteste rarement les droits « non nommés » dont bénéficie le groupe majoritaire anglophone et blanc. En d'autres mots, promouvoir le multiculturalisme semble le plus facile dans une situation où l'on peut faire référence aux droits «explicites » (réclamés et en partie octroyés) des minorités nationales. Le nationalisme majoritaire camouflé (Lecours et Nootens, 2007), par contre, n'offre guère de point d'attaque pour des revendications pluralistes. En revanche, le multinationalisme explicite permet au multiculturalisme d'être inséré comme une extension - plutôt que comme une rupture - de la « tradition ». La prolifération de la pluralité ethnique devient ainsi un élargissement du compromis pluraliste antérieur : elle favorise l'idée de la nation pluraliste plus qu'elle ne la fait échouer. Selon cette logique, le multiculturalisme embellit et complète le multinationalisme.

La similarité qui est construite entre les « groupes ethniques » immigrés et la (les) nation(s) minoritaire(s) comporte aussi des inconvénients. En cas de conflit, le lien entre le multinationalisme et le multiculturalisme peut être facilement renversé. Lorsque les membres des groupes établis n'arrivent pas à s'accorder sur les privilèges respectifs, les « problèmes » que pose le nationalisme minoritaire sont parfois projetés sur les minorités ethniques. L'idée d'une similitude logique entre le multinationalisme et le multiculturalisme persiste, mais, elle est cette fois utilisée pour contester tout type de droits collectifs. Que les traits négatifs attribués aux anciennes minorités nationales puissent être facilement élargis aux nouvelles minorités issues de l'immigration montre que le multinationalisme, en tant que compromis historiquement établi, ne détermine pas les attitudes envers l'adaptation et l'intégration des nouveaux venus. Aucun compromis n'est toutefois unanime. Si un parallèle entre anciennes et nouvelles minorités est établi, les adversaires du compromis pluraliste peuvent aussi instrumentaliser cette similitude.

Pour contester la thèse selon laquelle la reconnaissance de la diversité ethnique conduit nécessairement à la fragmentation sociétale, le multiculturalisme a été récemment légitimé non pas par rapport à sa similarité avec le multinationalisme mais, par opposition à celui-ci. Comparées à la quête d'autonomie politique et de séparation des minorités nationales, les demandes de droits «polyethniques » et « de représentation » que formulent les groupes ethniques, selon les termes de Kymlicka (1995), apparaissent plutôt inoffensives. Dans cette logique, les représentations du multiculturalisme ne déplacent, ni ne font voler en éclats, l'idée d'une (seule) nation 
multiculturelle. Alors que le multinationalisme devient marginalisé à cause du potentiel de conflit qui lui est inhérent, les minorités ethniques, dans cette perspective, sont de plus en plus présentées comme de «véritables » porteurs de la diversité. Leur « multiculturalisme » confère à l'identité nationale une signification car il est situé socialement entre (comme « ciment») et moralement au-dessus (comme «face visible » de la nation) des « nations fondatrices » querelleuses.

Au Canada, dans les années 1990, deux événements ont contribué à la dissociation entre multiculturalisme et fragmentation sociale. Premièrement, dans le contexte d'un conflit marqué entre les deux « nations fondatrices » canadiennes, que souligna le référendum de 1995 sur la souveraineté du Québec, la logique selon laquelle « les minorités ethniques sont semblables au Québec » commence à être remise en question. L'opposition au nationalisme minoritaire permet aux membres des groupes ethniques d'être représentés, et de se représenter eux-mêmes, comme de meilleurs citoyens. Compte tenu de leur adhésion à un projet d'identité nationale unifiée, les immigrants devraient être préférés aux ethnonationalistes ou encore aux séparatistes. Deuxièmement, la publication de Multicultural Citizenship (Kymlicka) dans la même année a fourni une légitimation scientifique puissante au multiculturalisme «non menaçant » des immigrants. Ainsi, les enjeux multinationaux et multiculturels de cette époque sont mieux décrits par l'hypothèse selon laquelle le conflit sur le multinationalisme dans les représentations publiques de «qui nous sommes » a facilité la reconnaissance des immigrants et de leurs enfants comme membres de la société majoritaire.

Notre analyse suggère, sans pour autant le prouver totalement, que le conflit entre les groupes nationaux a renforcé la légitimité des groupes ethniques issus de l'immigration. À notre avis, c'est une des raisons pour laquelle la nouvelle critique « anti-pluraliste » qui a gravement touché de nombreux pays européens depuis la fin des années 1990 (Bader, 2005) arrive plus tard et se manifeste plus faiblement au Canada (Kymlicka, 2004). En effet, le multiculturalisme revendiqué de la population immigrante au Canada semble plutôt individualiste, culturaliste et intégratif. Des chercheurs constatent un manque de résistance vis-à-vis de la réinterprétation successive du multiculturalisme en notion identitaire promue par l'État. Autant que la présence du multiculturalisme symbolique augmente sur la place publique, le pluralisme structurel - l'appui pour des associations « mono-ethniques » et des institutions séparées dans une même société - devient marginal (McAndrew et al., 2005). Il reste toutefois à déterminer, de façon critique, si l'intégration du multiculturalisme dans l'imaginaire de la société multinationale n'est qu'une stratégie dominante temporaire ou si elle produit des effets émancipateurs pour les groupes issus de l'immigration à long terme. Les débats récents autour de l'" accommodement raisonnable » des droits à la différence des minorités religieuses en constituent le nouveau défi. 


\section{Références Bibliographiques}

ABU-LABAN Yasmeen, GABRIEL, Christina (2002) Selling Diversity: Immigration, Multiculturalism, Employment Equity, and Globalization, Peterborough, Broadview Press.

ANCTIL Pierre (1984) «Double majorité et multiplicité ethnoculturelle à Montréal », Recherches sociographiques, 25, pp. 441-456.

ANDERSON Benedict ([1983] 1991) Imagined Communities: Reflections on the Origin and Spread of Nationalism, $2^{\mathrm{d}}$ ed., London, New York, Verso.

BADER Veit (ed.) (2005) « Multicultural Futures? International Approaches to Pluralism », Canadian Diversity/Diversité canadienne, 4 (1), 112 p.

BISSOONDATH Neil (1994) Selling Illusions: The Cult of Multiculturalism in Canada, Toronto, Penguin Books.

BOUTHILLIER Guy (1997) L'obsession ethnique (essai), Outremont, Lanctot, 238 p.

BRETON Raymond (1964) «Institutional Completeness of Ethnic Communities and Personal Relations of Immigrants », American Journal of Sociology, 70, pp. 193-205.

COMMISSION ROYALE POUR LE BILINGUISME ET LE BICULTURALISME (1969) Rapport Final, Ottawa, Supply and Services Canada, tome IV.

CORBO Claude (1996) Lettre fraternelle, raisonnée et urgente à mes concitoyens immigrants, Outremont, Lanctôt.

DROUILLY Pierre (1997) Indépendance et démocratie : sondages, élections et référendums au Québec, Montreal, L'Harmattan.

ELIAS Norbert, SCOTSON John ([1976] 1994) «Introduction: A Theoretical Essay on Established and Outsider Relations », The Established and the Outsiders. A Sociological Inquiry into Community Problems, London, Sage, pp. xv-lii.

FAIRCLOUGH Norman (1992) Discourse and Social Change, Cambridge, Polity Press.

FOUROT Aude-Claire (2006) « Le désalignement des politiques d'immigration et d'intégration au sein de 1'Union Européenne : Hardlaw versus Softlaw ? »Review of European and Russian Affairs, 2 (3), pp. 147-173.

GOVERNMENT OF CANADA (2004) The Speech from the Throne of February, Ottawa, Office of the Prime Minister, 2 février.

HELLY Denise (2004) «Are Muslims Discriminated Against in Canada Since September 2001? » Canadian Ethnic Studies, 36 (1), pp. 24-47.

HOUSE OF COMMONS (1971) Debates, Ottawa, Queen's Printer, 8 octobre.

JOPPKE Christian (2007) « Transformation of Citizenship: Status, Rights, Identity », Citizenship Studies, 11 (1), pp. 37-48.

JUTEAU Danielle (2000) «Du dualisme canadien au pluralisme québécois », in Marie McAndrew, France Gagnon Eds., Relations ethniques et éducation dans les sociétés divisées (Québec, Irlande du Nord, Catalogne et Belgique), Paris, Montreal, L'Harmattan, pp. 13-26.

KARIM Karim (2002) «The Multiculturalism Debate in Canadian Newspapers: The Harbinger of a Political Storm? », Journal for International Migration and Integration (RIMI), 3 (3 \& 4), pp. 439-455.

KELNER M., KALLEN E. (1974) «The Multicultural Policy: Canada's Response to Ethnic Diversity », Journal of Comparative Sociology, 2, pp. 21-34.

KYMLICKA Will (1995) Multicultural Citizenship: A Liberal Theory of Minority Rights, Oxford, Clarendon Press.

KYMLICKA Will (2003) «Being Canadian », Government and Opposition, 38 (3), pp. 357-385. KYMLICKA Will (2004) "Marketing Canadian Pluralism in the International Arena », International Journal, 59 (4), pp. 829-852. 
LECOURS André, NOOTENS Geneviève (2007) "Comprendre le nationalisme majoritaire », in Alain-G Gagnon et al. Eds., Les Nationalismes majoritaires contemporains : identité, mémoire, pouvoir, Montreal, Québec Amérique, pp. 19-45.

MÁIZ Ramón, REQUEJO Ferran (2005) «Introduction », in Ramón Máiz, Ferran Requejo Eds., Democracy, Nationalism and Multiculturalism, London, New York, Frank Cass, pp. 1-12.

MCANDREW Marie et al. (2005) "Pour un débat éclairé sur la politique canadienne du multiculturalisme: une analyse de la nature des organismes et des projets subventionnés (1983-2002) », Politique et sociétés, 24 (1), pp. 49-71.

MCROBERTS Kenneth (1997) Misconceiving Canada. The Struggle for National Unity, Toronto, Oxford University Press.

MILLS C. Wright ([1959] 2000) The Sociological Imagination, Oxford, New York, Oxford University Press.

NEU Dean, THERRIEN Richard (2003) Accounting for Genocide: Canada's Bureaucratic Assault on Aboriginal People, Black Point (NS), Fernwood Publishing.

PENNINX Rinus, SCHROVER Marlou (2002) « Bastion of bindmiddel? Organisaties van immigranten in historisch perspectief », in Jan Lucassen, Arie de Ruijter Eds., Nederland: Multicutureel en pluriform? Een aantal conceptuele studies, Amsterdam, Aksant, pp. 279322.

PFAFF-CZARNECKA Joanna (2004) « Diversity, Immigration, and National Identity in Switzerland », Canadian Diversity/Diversité canadienne, 3 (2), pp. 77-80.

PIETRANTONIO Linda et al. (1996) «Multiculturalisme ou intégration: un faux débat », in Khadiyatoulah Fall et al. Eds., Les convergences culturelles dans les sociétés pluriethniques, Quebec, Presses de 1'Université Laval, pp. 147-158.

RAZACK Sherene (2007) "The 'Sharia Law Debate' in Ontario: The Modernity/Premodernity Distinction in Legal Efforts to Protect Women from Culture », Feminist Legal Studies, 15 (1), pp. 3-32.

ROBERTSON ROLAND (1995) «Glocalization: Time-space and HomogeneityHeterogeneity », in Mike Featherstone, Scott Lash Eds., Global modernities, London, Sage, pp. 25-44.

ROCHER Guy (1971) «Les ambiguïtés d'un Canada bilingue et multiculturel », Revue de l'Association canadienne d'éducation de langue française, 1 (3), pp. 21-23.

SCHWIMMER Éric (1995) Le syndrome des Plaines d'Abraham, Montreal, Boréal.

SIMMONS Alan (2004) «Critical Reflections on Immigration and Labour Force Skill Levels: New Data and Emerging Frameworks », communication présentée dans le cadre de la conférence Gender and Work Database: Knowledge Production in Practice, Toronto, York University, 1-2 octobre.

STASIULIS Daiva (1995) «'Deep Diversity': Race and Ethnicity in Canadian Politics », in M. Whittington, G. Williams Eds., Canadian Politics in the 1990s, Toronto, Nelson Canada, pp. 191-217.

STOFFMAN Daniel (2002) Who Gets In? What's Wrong with Canada's Immigration Program and How to Fix It, Toronto, Macfarlane Walter \& Ross.

TAYLOR Charles (1993) Reconciling the Solitudes. Essays on Canadian Federalism and Nationalism, Montreal, Kingston, McGill-Queen's University Press.

VASTA Ellie (2007) «From Ethnic Minorities to Ethnic Majority Policy: Multiculturalism and the Shift to Assimilationism in the Netherlands », Ethnic and Racial Studies, 30 (5), pp. 713 740 .

WIMMER Andreas (2002) Nationalist Exclusion and Ethnic Conflict: Shadows of Modernity, Cambridge, Cambridge University Press.

WINTER Elke (2004) Max Weber et les relations ethniques. Du refus du biologisme racial à l'État multinational, Quebec, Presses de l'Université Laval. 
WINTER Elke (2007a) « How Does the Nation Become Pluralist? », Ethnicities, 7 (4), pp. 483515.

WINTER Elke (2007b) « Neither 'America' nor 'Québec': Constructing the Canadian Multicultural Nation », Nations and Nationalism, 13 (3), pp. 481-503.

WODAK Ruth et al. (1998) Zu diskursiven Konstruktion nationaler Identität, Frankfurt a.M., Suhrkamp. 


\title{
Les logiques du multiculturalisme dans les sociétés multinationales : une analyse des discours canadiens
}

\author{
Elke WINTER
}

S'inspirant de l'exemple canadien, cet article explore les relations entre le multinationalisme et le multiculturalisme au sein d'une même société. Si le premier concerne l'accommodement des minorités dites « nationales ", le deuxième s'adresse aux minorités ethniques d'origine immigrée, dont il vise l'intégration dans la société majoritaire. Bien qu'analytiquement distincts, ces deux mouvements sociaux et les politiques respectives sont interdépendants. Dans cet article, nous examinons les argumentations, puisées dans la presse canadienne, qui servent à légitimer ou à délégitimer le multiculturalisme d'origine immigrée en regard du nationalisme minoritaire québécois. Notre recherche nous permet d'identifier quatre scénarios selon la manière dont l'accommodement des groupes " nationaux » historiquement établis influe sur la mise en œuvre des politiques multiculturelles en réponse à une diversité récente, induite par l'immigration.

\section{The Logics of Multiculturalism in Multinational Societies: An Analysis of Canadian Discourses}

\section{Elke WINTER}

Taking its inspiration from the Canadian case, this paper explores the relationship between multinationalism and multiculturalism within society. Whereas the former addresses the accommodation of so-called 'national' minorities, the latter deals with ethnic minorities of immigrant origin and their integration into mainstream society. Although analytically different, these two social movements and their respective policies impact each other mutually. Drawing upon a study of English Canadian newspaper discourses, this paper examines whether and how representations of Québécois nationalism impact on legitimizations and delegitimizations of multiculturalism. The research reveals four templates of how historically established accommodation of national groups influences the rationale for implementing multicultural policies in response to recent diversity caused by immigration.

\section{Las lógicas del multiculturalismo en sociedades multinacionales: un análisis de los discursos canadienses}

\section{Elke WINTER}

Tomando como punto de partida la sociedad canadiense, este artículo explora la relación entre el multinacionalismo y el multiculturalismo. Mientas que por un lado el multinacionalismo se ocupa del reconocimiento institucional y simbólico de las llamadas minorías "nacionales"; el multiculturalismo por otro acomoda a las minorías "étnicas" inmigrantes y su integración en la sociedad general. Aún cuando sean analíticamente diferentes, estos dos movimientos sociales y sus respectivas políticas se condicionan e impactan mutuamente. Sobre la base de un estudio de los discursos en los periódicos canadienses de habla inglesa, este ensayo examina como las representaciones del nacionalismo quebequense impactan la legitimizacion y/o la deslegimizacion del multiculturalismo. La investigación revela cuatro modelos de cómo los grupos nacionales — históricamente consolidados — han influenciado la implementación de las políticas multiculturales que responden a la reciente diversidad cultural causada por los flujos migratorios. 


\section{tsantsa}

REVUE DE LA SOCIETE SUISSE D'ETHNOLOGIE

ZEITSCHRIFT DER SCHWEIZERISCHEN ETHNOLOGISCHEN GESELLSCHAFT

RIVISTA DELLA SOCIETA SVIZZERA D'ETNOLOGIA

\section{DOSSIER $132008 \cdot$ RAPPORTS DE POUVOIR - MACHTVERHÄLTNISSE}

Séverine Rey RAPPORTS DE POUVOIR - MACHTVERHÄLTNISSE

INTRODUCTION À UNE PERSPECTIVE ANTHROPOLOGIQUE - EINFÜHRUNG IN EINE ANTHROPOLOGISCHE PERSPEKTIVE

Véronique Mottier

THEORISING SEXUALITY

GENDER POWER, FEMINISM AND SEXUAL «LIBERATION»

Arnaud Esquerre QUAND LE PSYCHISME EST OBJET DE POUVOIR

LA MANIPULATION MENTALE COMME RAPPORT DE FORCE DANS LE CADRE DE LA LUTTE CONTRE LES «SECTES» EN FRANCE

Florence Graezer Bideau

Sabrina Beeler Stücklin

Yazid Ben Hounet

Sabine Kradolfer

Valerio Simoni

Carena Brenner

Brigit Allenbach

Simone Haug und Julia Weber

Reto Kuster

Vanessa Langer

Martin Sökefeld

Géraldine Morel-Baro Diaz

Barbara Muller

Eva Soom Ammann

REVUE ANNUELLE TSANTSA JAHRESZEITSCHRIFT TSANTSA

www.seg-sse.ch
Preis einer Ausgabe/Prix au numéro: 35- CHF (24 - Euro)

Abonnement für 3 Ausgaben / Abonnement pour 3 numéros: 95.- CHF (64.- Euro)

Bitte kontaktieren Sie / Veuillez contacter:

SEISMO VERLAG · Zähringerstrasse 26·CH-8001 Zürich · buch@seismoverlag.ch · www.seismoverlag.ch

\section{LE CAS DE LA DANSE DU YANGGE EN CHINE}

RESSOURCENDEGRADIERUNG UND KONFLIKTE

MACHTVERHÄLTNISSE UND INSTITUTIONELLER WANDEL IM NIGERBINNENDELTA VON MALI

YKHET EMIR

RAPPORTS HIÉRARCHIQUES ET RAPPORTS DE POUVOIR EN MILIEU TRIBAL

RÉSISTER DANS LA DIVISION

ARTICULATIONS ET DÉSARTICULATIONS DU POUVOIR CHEZ LES MAPUCHE DE NEUQUÉN (ARGENTINE)

SHIFTING POWER

THE (DE)STABILIZATION OF ASYMMETRIES IN THE REALM OF TOURISM IN CUBA

FILM ALS KRITIK AM FILM

ARTICLES LIBRES - FREIE BEITRÄGE

«ICH BIN IN EINER ZWICKMÜHLE»

MIGRATION, VERWANDTSCHAFT UND GESCHLECHT AM BEISPIEL DER ALBANISCHEN DIASPORA IN DER SCHWEIZ

ESSAIS EN ANTHROPOLOGIE VISUELLE - BILD-ESSAYS

DURCHGANGSZENTRUM

RÄUMLICHKEITEN UND GRENZZIEHUNGEN

PHOTOGRAPHY IN THE ITURI FOREST (DEMOCRATIC REPUBLIC OF THE CONGO)

REGARDS SUR LE VOILE

UNE APPROCHE RÉFLEXIVE SUR LE PROCESSUS DE FABRICATION DES IMAGES DE MON PREMIER FILM DOCUMENTAIRE

MOVING IMAGES

TRUCK ART IN PAKISTAN

RECHERCHES EN COURS - LAUFENDE FORSCHUNGEN

PÈRES ET CHEIKHS

RAPPORTS D'ÂGE ET D'AUTORITÉ RELIGIEUSE AU CAIRE

PUISSANCES ABAKUÁS ET POUVOIR POLITIQUE À LA HAVANE

ENTRE INSTITUTIONNALISATION ET CONTESTATION

RÉFLEXIONS ANTHROPOLOGIQUES SUR LES MÉDICAMENTS

UN TERRAIN «TRĖS PROCHE»

ALTERN ALS PAAR IN DER MIGRATION

DIE SOLIDARGEMEINSCHAFT EHE

ISBN-13: 978-3-03777-013-9/ISBN-10: 3-03777-013-9/ISSN: 1420-7834 